\title{
Positron annihilation spectroscopy study of lattice defects in non-irradiated doped and un-doped fuels
}

\author{
Mélanie Chollet*, Vladimir Krsjak, Cédric Cozzo, and Johannes Bertsch \\ Nuclear Energy and Safety Department, Paul Scherrer Institut, 5232 Villigen PSI, Switzerland
}

Received: 23 September 2015 / Received in final form: 17 June 2016 / Accepted: 5 December 2016

\begin{abstract}
Fission gas behavior within the fuel structure plays a major role for the safety of nuclear fuels during operation in the nuclear power plant. Fission gas distribution and retention is determined by both, micro- and lattice-structure of the fuel matrix. The ADOPT (Advanced Doped Pellet Technology) fuel, containing chromium and aluminum additives, shows larger grain sizes than standard (undoped) $\mathrm{UO}_{2}$ fuel, enhancing the fission gas retention properties of the matrix. However, the additions of such trivalent cations shall also induce defects in the lattice. In this study, we investigated the microstructure of such doped fuels as well as a reference standard $\mathrm{UO}_{2}$ by positron annihilation spectroscopy (PAS). Although this technique is particularly sensitive to lattice point defects in materials, a wider application in the $\mathrm{UO}_{2}$ research is still missing. The PAS-lifetime components were measured in the hotlab facility of PSI using a ${ }^{22} \mathrm{Na}$ source sandwiched between two $500-\mu \mathrm{m}-$ thin sample discs. The values of lifetime at the center and the rim of both samples, examined to check at the radial homogeneity of the pellets, are not significantly different. The mean lifetimes were found to be longer in the ADOPT material, $220 \mathrm{ps}$, than in standard $\mathrm{UO}_{2}, 190 \mathrm{ps}$, which indicates a larger presence of additional defects, presumably generated by the dopants. While two-component decomposition (bulk + one defect component) could be performed for the standard material, only one lifetime component was found in the doped material. The absence of the bulk component in the ADOPT sample refers to a saturated positron trapping (i.e., all positrons are trapped at defects). In order to associate a type of lattice defect to each PAS component, interpretation of the PAS experimental observations was conducted with respect to existing experimental and modeling studies. This work has shown the efficiency of PAS to detect lattice point defects in $\mathrm{UO}_{2}$ produced by $\mathrm{Cr}$ and $\mathrm{Al}$ oxides. These additives create lattice irregularities, which are acting as sinks for fission products on one hand and trapping positrons on the other hand. Fitting of the obtained experimental data with a suitable theoretical model can provide a valuable qualitative assessment of these defects. At this stage of the research, some of the existing models were used for this purpose.
\end{abstract}

\section{Introduction}

It is well established that the addition of chosen dopants in $\mathrm{UO}_{2}$ fuel, the most popular being $\mathrm{Cr}_{2} \mathrm{O}_{3}$, enlarges grain sizes contributing to a better fission gas retention and improves pellet-cladding interaction behavior [1]. While the oxidation state of $\mathrm{Cr}$ has been recently assessed to be +3 only [2], the mechanism of accommodation of such cation in the face centered cubic (f.c.c.) structure of the $\mathrm{UO}_{2}$ is still not entirely understood: is $\mathrm{Cr}$ accommodated in substitution? Of oxygen? Uranium? In interstitials? In vacancies or clusters of vacancies already present in the lattice? Whatever the mechanism, dopants are likely to induce point defects. In this study, we have investigated the occurrence of such lattice defects by positron annihilation spectroscopy (PAS).

\footnotetext{
* e-mail: melanie.chollet@psi.ch
}

PAS is a powerful technique to probe defects and has already widely been used for nuclear structural materials $[3,4]$. However, the number of published works on $\mathrm{UO}_{2}$ is small. Even less papers have addressed the issue of radiation effects [5-9] and there is only one study on doped-material by PAS where dopants were actinides [10]. The present study focuses for the first time on PAS characterization of $\mathrm{UO}_{2}$ fuel with a microstructure modified by dopants.

\section{Experimental}

The doped $\mathrm{UO}_{2}$ ADOPT (Advanced Doped Pellet Technology) and conventional $\mathrm{UO}_{2}$ Standard Optima2 (Std Opt2) fuels manufactured under similar conditions by Westinghouse (Västeras, Sweden) have been investigated in this study. Details of the fabrication process are given in 

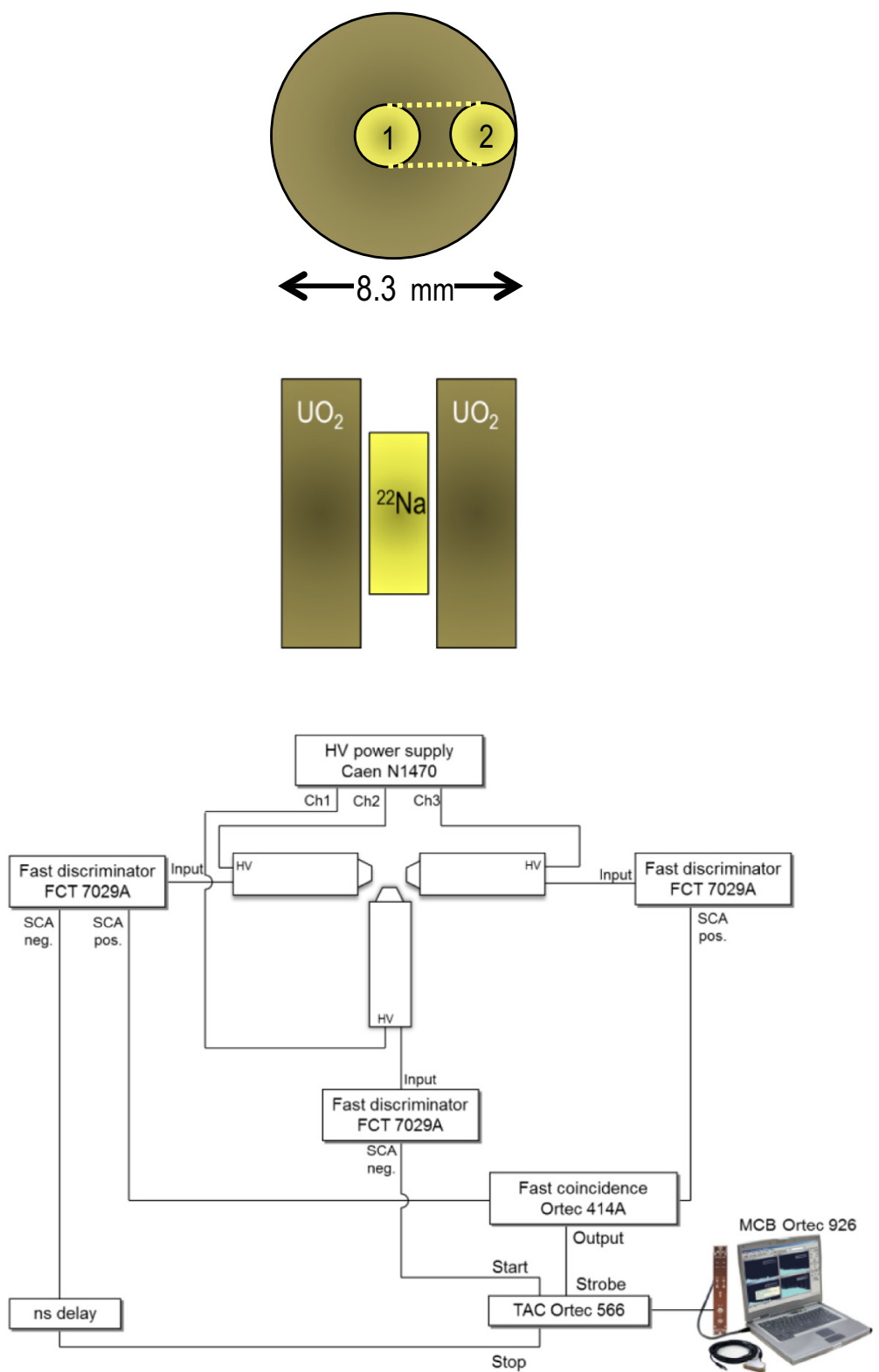

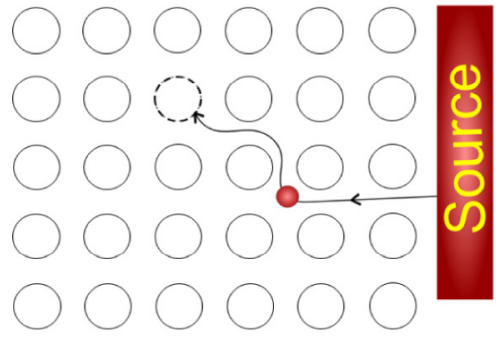

Positron lifetime spectroscopy - PALS ( $\beta^{+}$trapping)
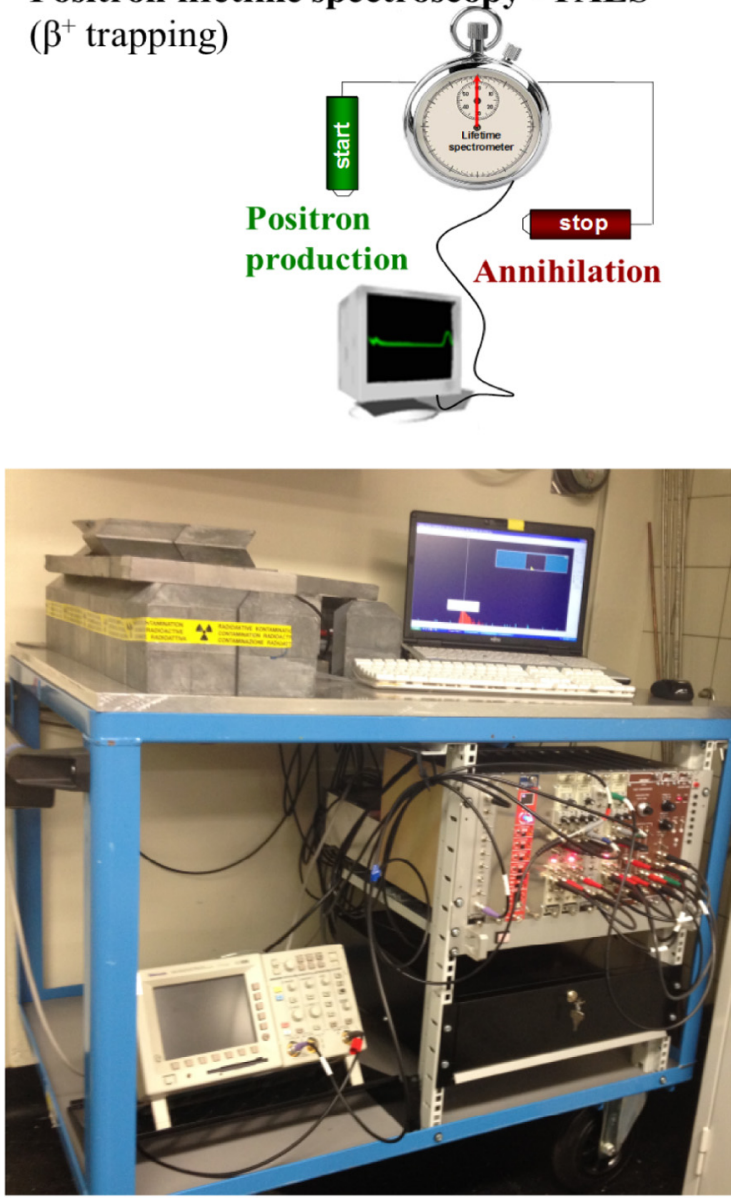

Fig. 1. Schematic drawings of the experimental setup (top and bottom left) and picture of the positron lifetime spectrometer facility (right). In this case, the spectrometer was used in 2-detector mode [3].

Arborelius et al. [11]. Both initial powders were pressed into green pellets with a force of $50 \mathrm{kN}$ and then sintered in a $\mathrm{H}_{2} / \mathrm{CO}_{2}$ gas mixture at $1800^{\circ} \mathrm{C}$ during $14 \mathrm{~h}$. Additives of $\mathrm{Cr}$ and $\mathrm{Al}$ are limited to $1000 \mathrm{ppm}$ in the ADOPT material. The pellet densities are respectively 10.67 and $10.60 \mathrm{~g} / \mathrm{cm}^{3}$, corresponding to $97.3 \%$ and $96.7 \%$ of the theoretical values, showing the effect of additives.

For both materials, two thin slices of the pellets of $8.36 \mathrm{~cm}$ in diameter were cut and one face polished to obtain discs of $500 \mu \mathrm{m}$ thickness.

We used the decay of ${ }^{22} \mathrm{Na}$ generating positrons as a source, described as following: ${ }^{22} \mathrm{Na} \rightarrow{ }^{22} \mathrm{Ne}+\beta^{+}+\nu_{\mathrm{e}}+\boldsymbol{\gamma}$. This source of 3.7 MBq, obtained from an evaporated drop of aqueous solution containing ${ }^{22} \mathrm{Na}$ salt, has an effective diameter of $2 \mathrm{~mm}$ and is embedded between Kapton foils. Thanks to the small size of the source relative to the pellet slices, two separate measurements could be performed, i.e., in the center and at the rim of the pellets to investigate the radial homogeneity. The source is sandwiched between the two pieces of each sample and detectors are placed at each side of the set-up (Fig. 1).

The positron lifetime measurements were performed using a conventional two-detector spectrometer with a resolution of 195 ps. Contribution of positrons annihilating within the source was determined by calibration measurements to have $20 \%$ intensity and 390 ps lifetime. A typical lifetime spectrum, as obtained for the both $\mathrm{UO}_{2}$ materials, calibration Fe sample and calibration ${ }^{60} \mathrm{Co}$ source, can be 


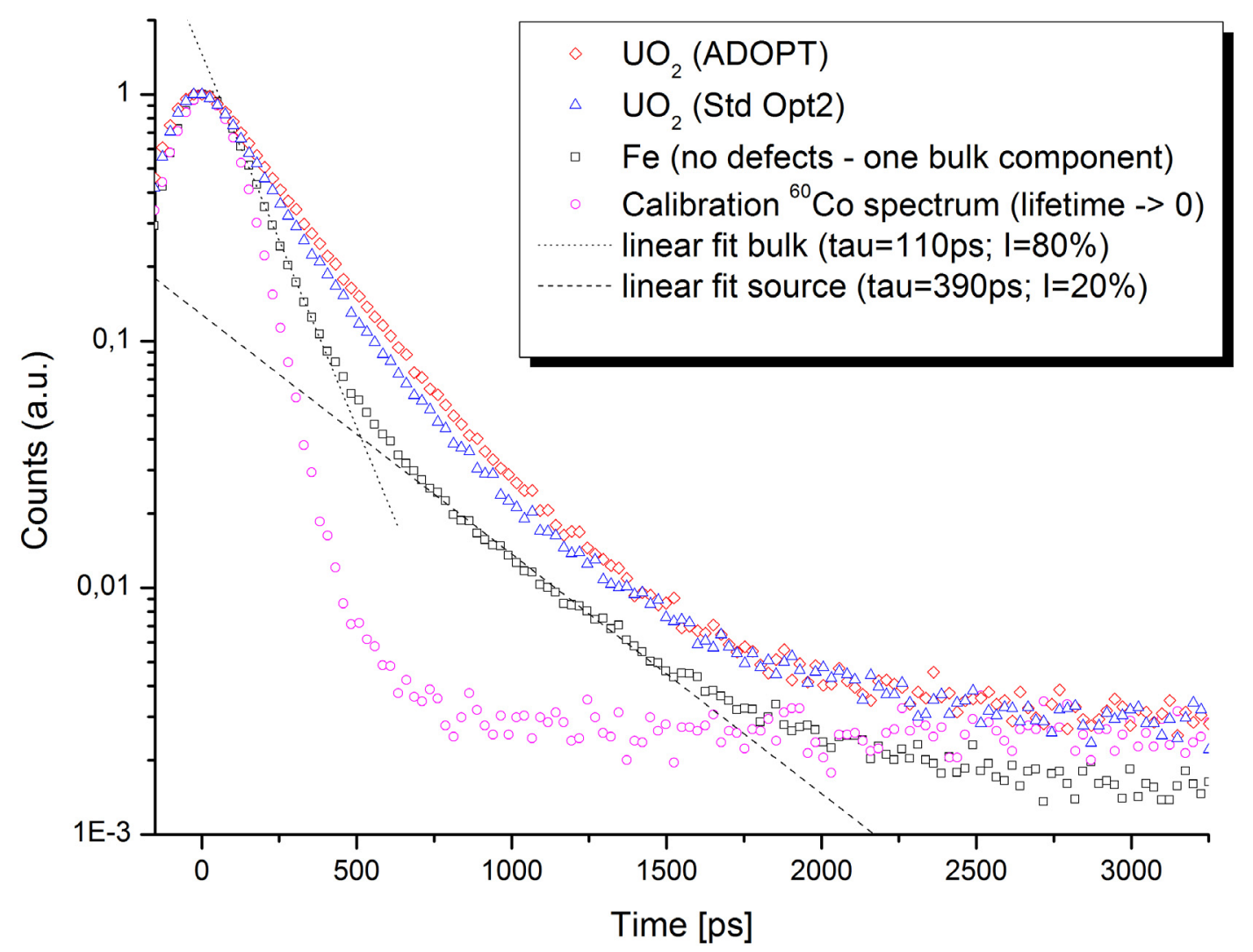

Fig. 2. Positron lifetime spectrum of the two investigated $\mathrm{UO}_{2}$ materials, defect-free Fe sample and calibration ${ }^{60} \mathrm{Co}$ source.

seen in Figure 2. A minor uncertainty could be introduced by not considering the effect of backscattered positrons in the calibration Fe sample. This uncertainty was considered negligible due to using of "fast" unmoderated positrons from radioisotope source.

\section{Results}

Analytical data processing was performed using the LT 9.0 program [12] and two-component decomposition of the spectra (bulk + defect component) according to the standard trapping model [13]. The lifetime spectra were fitted with a variance of fit (FV) ranging better than 1.06 (Tab. 1). In the case of the ADOPT sample, the bulk component could not be identified, which means that the positron trapping at defects reached its saturation (i.e., all positrons trapped). All experimental data are listed in Table 1. Two different values (250 and $300 \mathrm{ps}$ ) fixed for the defect component have been selected based on the previously published studies $[9,10]$ to examine scenarios with different types of defects.

Mean lifetimes are homogeneous at the center and the rim of the pellet at 190 and $220 \mathrm{ps}$ for both materials ADOPT and Std Opt2, respectively. It means that the microstructure along the pellet radius is not affected by the production process from a point defect perspective. Both pellets are radially isotropic. Mean lifetimes were found to be higher in the ADOPT material than in Std Opt2, indicating a higher number of point defects in the doped material. This is very likely due to the incorporation of trivalent cations $\left(\mathrm{Cr}^{3+}\right)$ in the structure.

As mentioned above, the spectra have been decomposed into two lifetimes $\tau_{1}$ and $\tau_{2}$. Two lifetime components are generally reported for $\mathrm{UO}_{2}[5,9,10]$. Such decomposition enables to calculate the lattice lifetime $\tau_{\text {bulk. }}$. For the Std Opt2 sample, $\tau_{\text {bulk }}=180 \mathrm{ps}$ was obtained. The first measured component $\tau_{1}$ of the Std Opt2 sample is by $10 \mathrm{ps}$ slightly lower than $\tau_{\text {bulk }}$, which is in agreement with the standard trapping model. This component could correspond either to a reassessment of a defect-free bulk or a mix of bulk and some shallow defects. In addition to this component, $15-33 \%$ of the positrons are trapped in defects with a lifetime of $250-300$ ps. The nature of this trapping site will be discussed in the next section. Larger defect structures as clusters with higher lifetimes (e.g., porosity) are not observed in either of the samples.

In the ADOPT fuel, positrons are trapped at defects (saturated positron trapping). Table 1 shows also some proposed fits with a fixed $\tau_{2}$ value at 250 or $300 \mathrm{ps}$. As can be seen, these fits result in a significantly reduced intensity of this component (as compared to the Std Opt2 sample) and suggest that such defects, are present in lower concentration than in the reference material or, if existent, are less attractive to positrons (so called shallow traps). 
Table 1. Mean lifetimes $\tau$, first and second components $\tau_{1}$ and $\tau_{2}$ and their associated intensity $I_{i}$. Italic values of $\tau_{2}$ indicate fixed parameters during the decomposition. Reduced chi-squares F.V. are given for each fit. Bulk lifetimes were calculated from the experimentally measured data according to the standard trapping model [13].

\begin{tabular}{llllllll}
\hline Sample & Mean $\tau(\mathrm{ps})$ & $\tau_{1}(\mathrm{ps})$ & $I_{1}(\%)$ & $\tau_{2}(\mathrm{ps})$ & $I_{2}(\%)$ & F.V. & $\tau_{\text {bulk }}(\mathrm{ps})$ calc. \\
\hline Std Opt2 center & 188 & 159 & 69.10 & 252 & 30.90 & 1.0315 & 179 \\
& 189 & 170 & 85.13 & 300 & 14.87 & 1.0318 & 182 \\
Sdt Opt2 rim & 189 & 158 & 66.30 & 250 & 33.70 & 1.0217 & 180 \\
& 191 & 171 & 84.78 & 300 & 15.22 & 1.0305 & 183 \\
ADOPT center & 218 & 218 & 100 & & & & \\
& 218 & 208 & 75.50 & 250 & 24.50 & 1.0045 & \\
ADOPT rim & 218 & 213 & 94.00 & 300 & 6.00 & 1.0035 & \\
& 221 & 221 & 100 & & & 1.0349 & \\
& 222 & 218 & 88.50 & 250 & 11.50 & 1.0673 & \\
\hline
\end{tabular}

\section{Discussion - interpretation of the PAS components}

The PAS signals of the standard and doped material are material-specific and indicate different microstructures. The few PAS-studies on $\mathrm{UO}_{2}$ material are too limited to establish a straightforward identification of the nature of the positron-trapping sites. In this section, we compare and discuss the results in the perspective of the previous experimental and modelling studies $[5,7,9,10]$. It is worth noting that these three available valuable studies stem from the same research group (CEA/CNRS, France). In particular, Wiktor et al. [9] have performed DFT $+U$ calculations to obtain the positron lifetimes of uranium and oxygen vacancies in $\mathrm{UO}_{2}$ as well as combination of vacancies (Shottky defect, etc.). They did not consider interstitial defects in their calculations.

The value of the lattice $\tau_{\text {bulk }}$ of $180 \mathrm{ps}$ compares well with the previous experimental values of $170-180 \mathrm{ps}$ in Roudil et al. [10] and $169 \mathrm{ps}$ in Barthe et al. [5]. It is possible that our value is slightly enhanced by a polishing effect or other intrinsic defects as no annealing was performed prior to measurements. Roudil et al. noticed a reduction of the bulk component from 180 to 170 ps for increasing annealing temperature, showing hence the recovering and removal of the bulk defects in the materials.

As already evoked, the $\tau_{1}$ at $170 \mathrm{ps}$ component in the standard material is probably a mix of the bulk component lowered by oxygen interstitial which is the most stable interstitial defect in the structure.

The $\tau_{2}$ component is higher than the mean value $\tau$, indicating positron trapping at vacancy-type defects (e.g., [9]). Several interpretations are possible for this component, but there is a consensus in the experimental studies $[5,10]$ to attribute the annihilation time between 250 and 300 ps to a displacement of U atoms (U-vacancies). The test-fits for the PAS signal with these imposed and fixed values at 250 and $300 \mathrm{ps}$ as $\tau_{2}$ component give good results given the FV values (Tab. 1). However, the formation energy of a uranium vacancy is almost twice that of oxygen [14], and in the literature this kind of point defect was generally detected in irradiated/damaged $\mathrm{UO}_{2}[5,10]$. In our fresh non-irradiated sample, the mechanism of creation of such defects could again be polishing, as already proposed by Evans et al. [7]. On the other hand, this 2 nd lifetime component $\tau_{2}$ is only observed in the standard sample, whereas both standard and doped samples have been polished; thus it should have also been detectable in the doped sample. Other mechanisms of formation are likely (e.g., intergranular misfits). Nevertheless, one can notice that fits including a fixed $\tau_{2}$ at 250 or 300 ps for the doped fuel data yield better or comparable variance of fit $\mathrm{FV}$ than those without $\tau_{2}$ (Tab. 1), so that the possibility of the occurrence of such U-vacancies should not be excluded in the doped material either. Other types of vacancies could also correspond to this $\tau_{2}$ component. Wiktor et al. determined that the well-stable Schottky defect $\left(\mathrm{V}_{\mathrm{U}}+2 \mathrm{~V}_{\mathrm{O}}\right)$ (neutral charge) shows lifetimes varying between 301 and $316 \mathrm{ps}$ depending of the lattice direction arrangement. Moreover, their energies of formation calculated by GGA $+U$ at $4.2 \mathrm{eV}$ are comparable to the one of oxygen vacancies [15], such that these defect clusters could also be considered for the $\tau_{2}$ lifetime component.

The nature of the lifetime at $220 \mathrm{ps}$ recorded for the doped material is more disconcerting than the one found in the standard sample, first because the trapping sites capture the totality of positrons up to saturation, second because this value was never reported in previous studies as a specific lifetime component. This component could be a signature of the defects created by the incorporation of additives in the $\mathrm{UO}_{2}$ lattice. Indeed, Riglet-Martial et al. [2] have shown by X-ray absorption near edge structure (XANES) that the oxidation state of soluble $\mathrm{Cr}$ is $3+$ only in $\mathrm{UO}_{2}$, creating obvious charge defects. According to the experimental and calculation work of Cardinaels et al. [16], the most favorable site for $\mathrm{Cr}$ satisfying the observed variation of lattice parameter of doped $\mathrm{UO}_{2}$ is the substitution of uranium combined with a bonding with a 
$\mathrm{U}^{+5}$ or one oxygen vacancy in neutral cluster. Oxygen vacancies, the most stable point defects in stoichiometric $\mathrm{UO}_{2}[9]$, are formally expected to be positively charged, and should therefore in principle be invisible to PAS. However, Vathonne et al. [15] has shown by DFT $+U$ method that $\mathrm{V}_{\mathrm{O}}$ charged -2 could also be stable for Fermi levels lying close to the middle of the band gap, so that the presence and detection of this very-stable defect should not be absolutely excluded. In order to provide a solid interpretation of the experimental data, more theoretical calculations are needed. Recently, collaboration with the Institute of Nuclear and Physical Engineering, Slovak University of Technology was established aiming to obtain an accurate theoretical interpretation.

\section{Conclusion and perspectives}

The PAS technique obviously highlights the microstructural particularity of doped vs. undoped $\mathrm{UO}_{2}$. We identified the bulk lifetime at $180 \mathrm{ps}$ in a quite good agreement with previous studies. A second component most probably corresponding to either U-vacancies or Schottky defects has been detected in the undoped material. In the ADOPT $\mathrm{UO}_{2}$, the defects created by the addition of dopants lead to a strongly localized trapping sites up to saturation. If the origin of this 220 ps component remains unclear, the PAS signal evidences the specific lattice particularities of this material. A modeling work using DFT $+U$ approach is ongoing in order to support assumptions and interpretation of the PAS signal. First calculations on $4 \times \mathrm{UO}_{2}$ supercell for $\mathrm{U}$-vacancy result in a good accordance with the present interpretation. The effect of $\mathrm{Cr}^{+3}$ incorporation will be studied in larger supercell $\left(32 \times \mathrm{UO}_{2}\right)$.

We believe this technique, up to now scarcely used for nuclear fuel, provides new valuable data on the $\mathrm{UO}_{2}$ latticemicrostructure and can be used as a quality assessment tool for fresh fuel. This is of particular interest, as the doped fuel seemingly exhibits a structural contradiction, i.e., higher general density (i.e., less pores), but also a higher density of point defects. However, the one does not exclude the other; and the higher density is beneficial for the thermo-physical properties whereas the point defects are trapping sites for volatile fission products (i.e., fission gas) atoms. The point defects quantification is well accessible by PAS. Unfortunately, although the analysis of defects in irradiated fuel would be of utmost interest, today PAS cannot be used for this case due to the strong activity of the fuel which affects the detector.
The authors are very grateful to the financial support of swissnuclear and Westinghouse for providing the samples. The authors also would like to acknowledge useful discussions with Eva Vitkovska and Peter Ballo from the Institute of Nuclear and Physical Engineering, Slovak University of Technology as well as with Claude Degueldre from PSI. Finally they deeply appreciate the sample preparation performed by Andrej Bullemer, PSI.

\section{References}

1. L. Bourgeois, P. dehaudt, C. Lemaignan, A. Hammou, J. Nucl. Mater. 297, 313 (2001)

2. C. Riglet-Martial, P. Martin, D. Testemale, C. SabathierDevals, G. Carlot, P. Matheron, X. Iltis, U. Pasquet, C. Valot, C. Delafoy, R. Largenton, J. Nucl. Mater. 447, 63 (2014)

3. V. Krsjak, J. Kuriplach, T. Shen, V. Sabelova, K. Sato, Y. Dai, J. Nucl. Mater. 456, 382 (2015)

4. V. Krsjak, V. Grafutin, O. Ilyukhina, R. Burcl, A. Ballesteros, P. Hähner, J. Nucl. Mater. 421, 97 (2012)

5. M.-F. Barthe, H. Labrim, A. Gentils, P. Desgardin, C. Corbel, S. Esnouf, J.P. Piron, Phys. Status Solidi C 4, 3627 (2007)

6. N. Djourelov, B. Marchand, H. Marinov, N. Moncoffre, Y. Pipon, P. Nédélec, N. Toulhoat, D. Sillou, J. Nucl. Mater. 432, 287 (2013)

7. H.E. Evans, J.H. Evans, P. Rice-Evans, D.L. Smith, C. Smith, J. Nucl. Mater. 199, 79 (1992)

8. H. Labrim, M.-F. Barthe, P. Desgardin, T. Sauvage, G. Blondiaux, C. Corbel, J.P. Piron, Appl. Surf. Sci. 252, 3256 (2006)

9. J. Wiktor, E. Vathonne, M. Freyss, G. Jomard, M. Bertolus, MRS Proc. 1645 (2014)

10. D. Roudil, M.F. Barthe, C. Jégou, A. Gavazzi, F. Vella, J. Nucl. Mater. 420, 63 (2012)

11. J. Arborelius, K. Backman, l. Hallstadius, M. Limbäck, J. Nilsson, B. Rebensdorff, G. Zhou, K. Kitano, R. Löfström, G. Rönnberg, J. Nucl. Sci. Technol. 43, 967 (2006)

12. J. Kansy, Nucl. Instrum. Methods Phys. Res. Sect. Accel. Spectrometers Detect. Assoc. Equip. 374, 235 (1996)

13. A. Vehanen, P. Hautojärvi, J. Johansson, J. Yli-Kauppila, P. Moser, Phys. Rev. B 25, 762 (1982)

14. B. Dorado, M. Freyss, B. Amadon, M. Bertolus, G. Jomard, P. Garcia, J. Phys. Condens. Matter 25, 333201 (2013)

15. E. Vathonne, J. Wiktor, M. Freyss, G. Jomard, M. Bertolus, J. Phys.: Condens. Matter 26, 325501 (2014)

16. T. Cardinaels, K. Govers, B. Vos, S. Van den Berghe, M. Verwerft, L. de Tollenaere, G. Maier, C. Delafoy, J. Nucl. Mater. 424, $252(2012)$ 